\title{
A GP1BA Variant in a Czech Family with Monoallelic Bernard-Soulier Syndrome
}

\author{
Magdalena Skalníková ${ }^{1,2, *(D)}$, Kateřina Staňo Kozubík ${ }^{1,2}$, Jakub Trizuljak ${ }^{1,2,3}$,, Zuzana Vrzalová ${ }^{1,2}$, \\ Lenka Radová ${ }^{1}\left(\mathbb{0}\right.$, Kamila Réblová ${ }^{1,2,3}{ }^{\oplus}$, Radka Holbová ${ }^{1}$, Terézia Kurucová ${ }^{1}$, Hana Svozilová ${ }^{1,2,3}{ }^{1}$, Jiři Štika ${ }^{1}$,

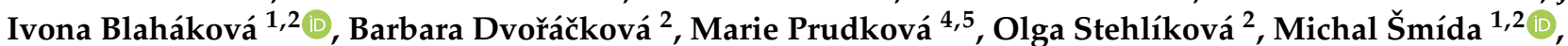 \\ Leoš Křen ${ }^{6}$, Petr Smejkal ${ }^{4,5}\left[\right.$, Šárka Pospíšilová ${ }^{1,2,3}$ and Michael Doubek ${ }^{1,2,3, * \mathbb{C}}$
}

check for updates

Citation: Skalníková, M.; Staňo

Kozubík, K.; Trizuljak, J.; Vrzalová, Z.; Radová, L.; Réblová, K.; Holbová, R.; Kurucová, T.; Svozilová, H.; Štika, J.; et al. A GP1BA Variant in a Czech Family with Monoallelic Bernard-Soulier Syndrome. Int. J. Mol. Sci. 2022, 23, 885. https:// doi.org/10.3390/ijms23020885

Academic Editor: Silvia S. Barbieri

Received: 30 November 2021

Accepted: 10 January 2022

Published: 14 January 2022

Publisher's Note: MDPI stays neutral with regard to jurisdictional claims in published maps and institutional affiliations.

Copyright: (C) 2022 by the authors. Licensee MDPI, Basel, Switzerland. This article is an open access article distributed under the terms and conditions of the Creative Commons Attribution (CC BY) license (https:// creativecommons.org/licenses/by/ $4.0 /)$.
1 Center of Molecular Medicine, Central European Institute of Technology, Masaryk University, 62500 Brno, Czech Republic; katerina.stanokozubik@ceitec.muni.cz (K.S.K.); trizuljak@gmail.com (J.T.); zuzana.vrzalova@ceitec.muni.cz (Z.V.); avodar@gmail.com (L.R.); kamila.reblova@ceitec.muni.cz (K.R.); radka.holbova@ceitec.muni.cz (R.H.); terezia.kurucova@ceitec.muni.cz (T.K.); svozilova.hana@gmail.com (H.S.); jiri.stika@ceitec.muni.cz (J.Š.); ivona.blahakova@ceitec.muni.cz (I.B.); michal.smida@ceitec.muni.cz (M.Š.); sarka.pospisilova@ceitec.muni.cz (Š.P.)

2 Department of Internal Medicine, Hematology and Oncology, University Hospital Brno and Faculty of Medicine, Masaryk University, 62500 Brno, Czech Republic; dvorackova.barbara@fnbrno.cz (B.D.); stehlikova.olga@fnbrno.cz (O.S.)

3 Institute of Medical Genetics and Genomics, University Hospital Brno and Faculty of Medicine, Masaryk University, 62500 Brno, Czech Republic

4 Department of Clinical Hematology, University Hospital Brno and Faculty of Medicine, Masaryk University, 62500 Brno, Czech Republic; prudkova.marie@fnbrno.cz (M.P.); smejkal.petr@fnbrno.cz (P.S.)

5 Department of Laboratory Methods, Faculty of Medicine, Masaryk University, 62500 Brno, Czech Republic

6 Department of Pathology, University Hospital Brno and Faculty of Medicine, Masaryk University, 62500 Brno, Czech Republic; kren.leos@fnbrno.cz

* Correspondence: magdalenaskalnikova@centrum.cz (M.S.); doubek.michael@fnbrno.cz (M.D.); Tel.: +421-54-949-8293 (M.S.)

Abstract: Bernard-Soulier syndrome (BSS) is a rare inherited disorder characterized by unusually large platelets, low platelet count, and prolonged bleeding time. BSS is usually inherited in an autosomal recessive (AR) mode of inheritance due to a deficiency of the GPIb-IX-V complex also known as the von Willebrand factor (VWF) receptor. We investigated a family with macrothrombocytopenia, a mild bleeding tendency, slightly lowered platelet aggregation tests, and suspected autosomal dominant (AD) inheritance. We have detected a heterozygous GP1BA likely pathogenic variant, causing monoallelic BSS. A germline GP1BA gene variant (NM_000173:c.98G > A:p.C33Y), segregating with the macrothrombocytopenia, was detected by whole-exome sequencing. In silico analysis of the protein structure of the novel GPIb $\alpha$ variant revealed a potential structural defect, which could impact proper protein folding and subsequent binding to VWF. Flow cytometry, immunoblot, and electron microscopy demonstrated further differences between p.C33Y GP1BA carriers and healthy controls. Here, we provide a detailed insight into its clinical presentation and phenotype. Moreover, the here described case first presents an mBSS patient with two previous ischemic strokes.

Keywords: Bernard-Soulier syndrome; monoallelic; autosomal dominant; GP1BA; macrothrombocytopenia

\section{Introduction}

Bernard-Soulier syndrome (BSS) is a rare inherited platelet disorder characterized by abnormally large platelets-macrothrombocytes-thrombocytopenia, and prolonged bleeding time, as well as with failure platelet aggregation after ristocetin [1-3]. BSS is caused by mutations in one of the following genes: GP1BA, GP1BB, or GP9. The mode of inheritance is usually AR (biallelic)—classical BSS. Clinically, patients with classical 
BSS present with a severe bleeding tendency, especially after events such as childbirth or surgery. Heterozygous carriers are usually asymptomatic and have normal platelet counts, although some might have slightly enlarged platelets and decreased platelet glycoprotein GPIb-IX-V complex expression, as well as a moderately reduced Ristocetin-Induced Platelet Aggregation (RIPA) response, a test triggering the binding of VWF to GPIb $\alpha[4,5]$. Moreover, rare variants in the GP1BA and GP1BB genes have been reported in patients with macrothrombocytopenia and the AD (monoallelic) mode of inheritance, presenting with a mild, usually asymptomatic form [3-12]. Around 12 missense mutations in the GP1BA gene have been described so far [4,8-15], one in a family from the Czech Republic. In addition, fourteen missense mutations in the GP1BB gene have been detected $[2,4-6,10]$. As GPIb $\alpha$ binds with other protein partners and influences primary hemostasis through several mechanisms, functional analysis of the aberrant protein is essential for confirmation of its pathogenicity [3-6].

\section{Results}

\subsection{Clinical Phenotype}

There were three patients suspected of inherited platelet function disorder (IPFD) and macrothrombocytopenia and two healthy individuals in the family investigated at the University Hospital Brno. Careful clinical evaluation of patients II-1 (proband), II-2, and III-1 (see Figure 1), including personal (healthy lifestyle) and familial bleeding history (unexplained bruising, epistaxis, menorrhagia, and bleeding during childbirth, and dental extractions) were obtained. Complete physical examination, such as hearing loss, heart, face, or bone dysmorphisms, ocular involvement, mental retardation, and skin discoloration was carried out. Furthermore, clinical assessment and a bleeding score were determined according to the bleeding questionnaire (Table S1) [16-18]. Total bleeding scores of the patients were evaluated as 6 (II-1), 5 (II-2), and 3 (III-1).

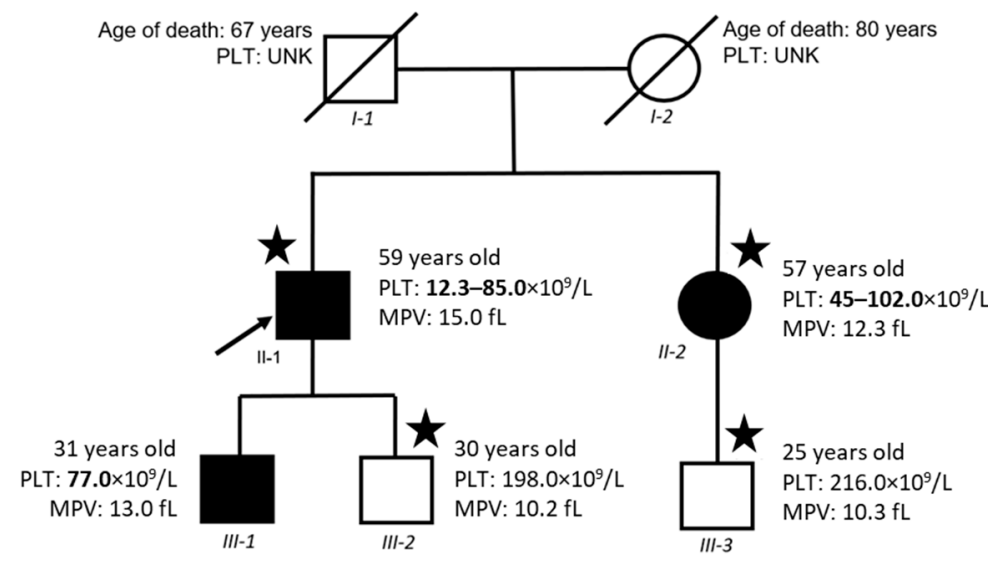

Figure 1. Proband's (II1) family pedigree shows segregation of macrothrombocytopenia, platelet count (PLT), and mean platelet volume (MPV). Squares represent male, circles represent female individuals, black squares and circles mean macrothrombocytopenia. The samples with an asterisk were analyzed by whole-exome sequencing. UNK-unknown.

In 2015, patient II-1 was first examined when he was admitted for a partial ischemic stroke of the left arterial circulation with secondary hemorrhagic changes, aphasia, and hemiparesis. At admission, the patient surprisingly was suffering from serious thrombocytopenia $\left(45 \times 10^{9} / \mathrm{L}\right)$. The patient underwent intensive physiotherapy and logotherapy. He received low-molecular-weight heparin (LMWH) at a prophylactic dose. Subsequently, LMWH was switched to low-dose acetylsalicylic acid (ASA). In 6 months, this patient was admitted again with a second ischemic stroke of the posterior cerebral circulation with paresis of the right lower limb. Antiplatelet therapy was switched from ASA to clopidogrel. Currently, this patient has recovered partially from the aphasia and hemiparesis. 
Regarding bleeding manifestations, patient II-1 suffered from repeated epistaxis with topical treatment in the past, bleeding from the gums only after brushing teeth, and prolonged post-traumatic bleeding. Unfortunately, light transmission aggregometry was first conducted after admission to the hospital on ASA treatment. In September 2017, the patient developed bradycardia $(45 / \mathrm{min})$ and paroxysm of atrial fibrilloflutter. Anticoagulant treatment was indicated. Further, a Boston Acclolade L311 DR pacemaker was implanted. For the next 3 months, the patient was treated with dual antiplatelet therapy and after that with ASA.

Patient II-2 underwent splenectomy with no bleeding complications. Splenectomy was indicated for steroid-refractory thrombocytopenia $\left(45 \times 10^{9} / \mathrm{L}\right)$. Consequently, there was an improvement in the platelet count after the splenectomy. Patients II- 1 and II- 2 underwent preliminary laboratory investigation, including full blood count and prothrombin time. Platelet counts and volume were controlled by counting platelets visually in a light microscope according to standard procedures. Platelet aggregation was performed in light transmission aggregometry (LTA) and the analysis of major platelet surface glycoproteins by flow cytometry (Figure 2). Whole-exome sequencing (WES) analysis was performed in two patients with macrothrombocytopenia (II-1; II-2) and two healthy individuals (III-2, III-3). Sanger sequencing confirmed the WES results and the absence of a GP1BA gene variant in the healthy individuals III-2 and III-3. In addition, the GP1BA variant was found by Sanger sequencing in the III-1 individual. Healthy individual III-3 was used as a control sample for flow cytometric analysis and transmission electron microscopy (TEM). Immunoblot of the platelet fraction in patient II-1 verified decreased expression of GPIb $\alpha$ compared with healthy individual III-2. Pedigree analysis indicated an AD mode of inheritance (Figure 1).

\subsection{Mutational Screening}

WES identified a heterozygous variant within GP1BA exon 2 (NM_000173:c.98G > A:p.C33Y) in both affected family members with macrothrombocytopenia (II-1; II-2). The variant was not detected in the healthy individuals (III-2, III-3). The results were verified by Sanger sequencing. The heterozygous variant within GP1BA exon 2 (NM_000173:c.98G $>$ A:p.C33Y) was also found in a third family member with macrothrombocytopenia (III-1) by Sanger sequencing.

First, the WES variants evaluation process was focused on the analysis of single nucleotide variants and short indels within the virtual panel of 363 platelet-associated genes (Table S4), where c.98G > A was characterized as the only "likely pathogenic" variant. In the next step, the evaluation of all WES data was performed, assuming the concordance of variants in both affected family members with regard to the mode of inheritance. Within the WES data, a total of 57 identical gene variants were identified in both patients, which were completely absent in healthy individuals (III-2, III-3) (Table S2). However, according to publications and databases, the detected gene variants, except for the GP1BA variant, have not yet been associated with inherited hematological diseases. Lastly, the presence of variants in 5'UTR of the ANKRD26 gene was screened by Sanger sequencing in five family members (II-1, II-2, III-1, III-2, III-3) with negative results. 

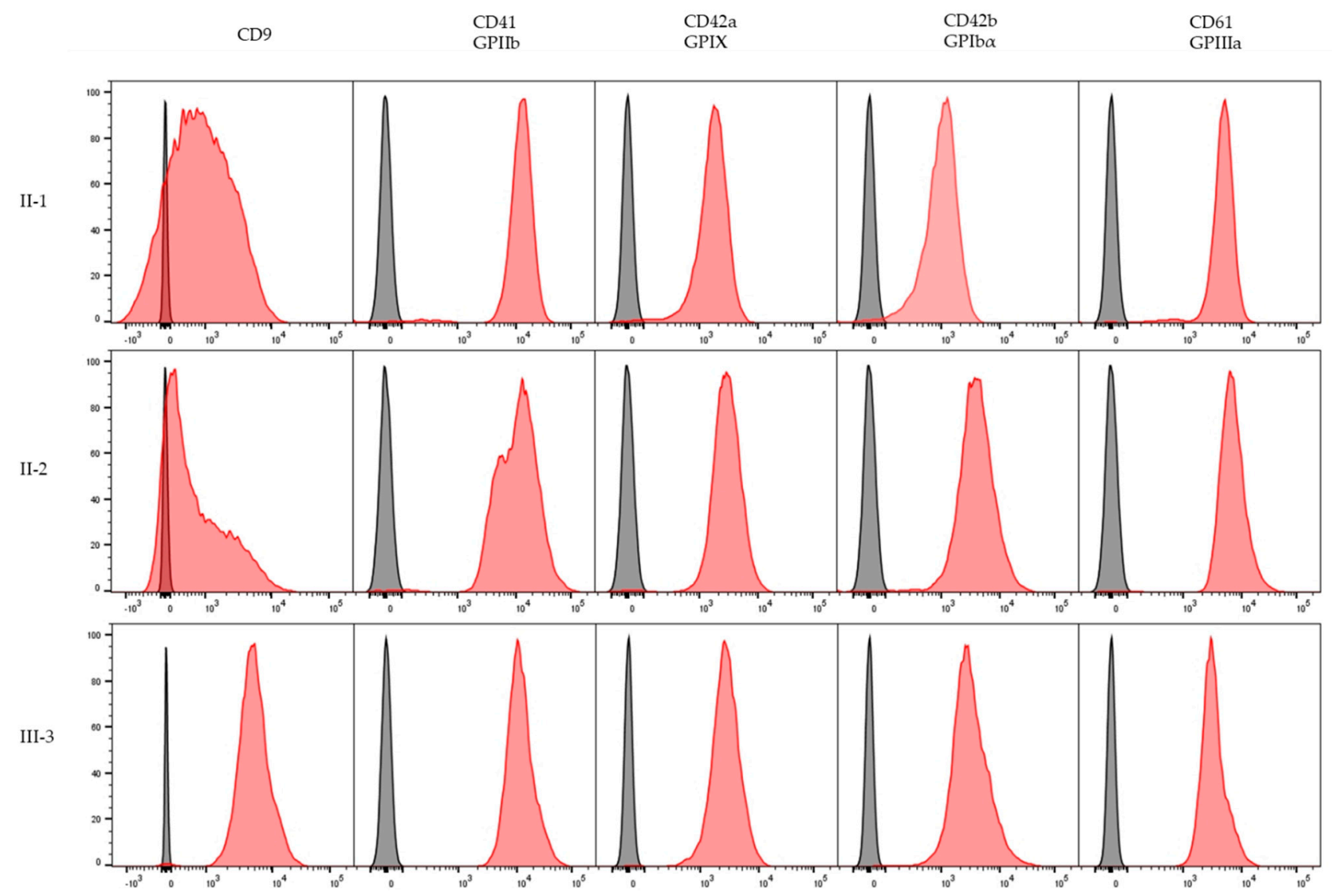

Figure 2. Platelet immunophenotype. CD41, CD61, CD42a, CD42b, CD9 antigens expression and negative controls on the platelets of two thrombocytopenic patients (II-1, II-2) and one healthy individual (III-3). Flow cytometric analysis shows decreased expression of the GPIb $\alpha$-IX complex and CD9 and slightly increased expression of GPIIb and GPIIIa in both patients compared to the healthy individual. Negative controls are in grey, population of interest is in red. The peak height is normalized to mode.

Recently, the single nucleotide substitution c.98G > A in the GP1BA gene was described in a Denmark family with diagnosed monoallelic BSS in three patients [10]. However, the variant c.98G > A is absent in ExAC, 1000 genomes, KAVIAR, gnomAD, HGMD databases, and Alamut ${ }^{\circledR}$ Visual 2.14 Software (SOPHiA Genetics, Boston, MA, USA). Ma et al. [11] in April 2021 reported a single case of an 18-month-old Chinese girl diagnosed with monoallelic BSS carrying a heterozygous c.97T > A variant in GP1BA which led to substitution p.C33R.

The GP1BA variant was located within the N-terminal leucine-rich repeat (LRR) domain of GPIb $\alpha$. The N-terminal domain of GPIb $\alpha$ consists of eight leucine-rich-repeats (LRRs) made up of parallel $\beta$-strands, which folds into an arc (Figure 3). The presence of central LRRs was suggested to be important for the binding of VWF [19]. Based on the crystal structure of the N-terminal domain of the human physiologic GPIb $\alpha$ [20], in silico analysis showed a disulfide bridge between Cys33 and Cys20. Replacement of cysteine 33 with tyrosine will disrupt this bridge, which most probably induces disruption of the protein structure. It is to be noted that Cys33 and Cys20 are localized in the loops adjacent to the terminal $\beta$ strands so they can contribute to the stability of the arc. Based on this knowledge, we presumed a pathogenic character of the p.C33Y variant. 


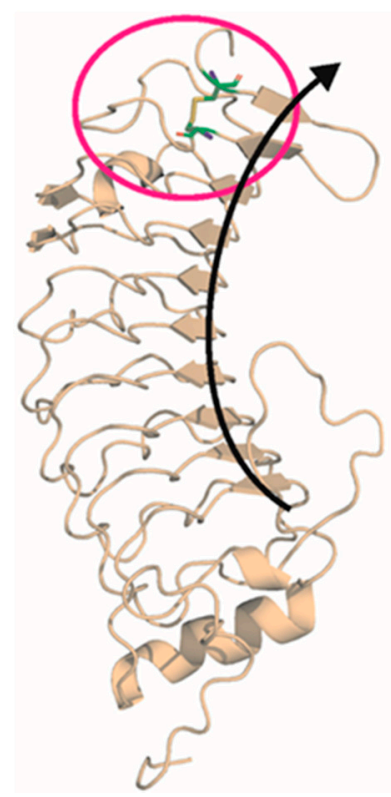

Figure 3. Human crystal structure of the N-terminal domain of GPIb $\alpha$ (pdb code 1p9a). Cys33 and Cys20 creating a disulfide bridge are highlighted in the magenta circle. Leucine-rich repeats folded into $\beta$-strands create an arc, which is highlighted by the black arrow. Cysteine-tyrosine substitution results in a disruption of a disulfide bond.

\subsection{Platelet Immunophenotype}

Flow cytometric analyses of expression of CD41 (GPIIb), CD61 (GPIIIa), CD42b $(\mathrm{GPIb} \alpha), \mathrm{CD} 42 \mathrm{a}$ (GPIX), and CD9 antigens in platelet-rich plasma (PRP) samples from the affected patients (II-1, II-2) and healthy individual (III-3) were performed. Platelet immunophenotype showed decreased expression of the GPIb $\alpha$-IX complex as well as CD9 in the patients and slightly increased GPIIb and GPIIIa expression (Figure 2) which corresponded with mean fluorescence intensity (MFI) values (Table S3). On the contrary, expression of all measured CD antigens was normal in a healthy individual (III-3).

\subsection{Platelet Aggregation Function}

The proband was receiving long-term antiplatelet therapy with acetylsalicylic acid (ASA) due to previous strokes. New platelet aggregation tests of the proband (II-1) revealed hypoaggregation $(60 \%)$ after ristocetin $(1.5 \mathrm{mg} / \mathrm{mL})$; however, there was also significant hypoaggregation after other platelet agonists: adenosine diphosphate (ADP, $5 \mu \mathrm{mol} / \mathrm{L}$ and $10 \mu \mathrm{mol} / \mathrm{L}$ ), collagen $(2 \mu \mathrm{g} / \mathrm{mL}$ and $5 \mu \mathrm{g} / \mathrm{mL}$ ), and arachidonic acid (ARA, $1.5 \mathrm{mmol} / \mathrm{L}$ ). The platelet aggregation function was normal in the healthy individual III-2 (Table 1). Original platelet aggregation tests (2017) of the proband (II-1) and his sister (II-2) revealed severe hypoaggregation $(20 \%)$ after ristocetin $(1.5 \mathrm{mg} / \mathrm{mL})$; however, there was also significant hypoaggregation after other platelet agonists (Table S5). 
Table 1. The reference limits and maximum aggregation results in patient II-1 and healthy individual III-2.

\begin{tabular}{|c|c|c|c|}
\hline Name of Agonists & Reference Limits & Patient II-1 & Healthy Individual III-2 \\
\hline Platelet count in PRP $\left(\times 10^{9} / \mathrm{L}\right)$ & $150-300$ & 105 & 228 \\
\hline Platelet count in $\mathrm{PPP}\left(\times 10^{9} / \mathrm{L}\right)$ & $0-20$ & 0 & 1 \\
\hline \multicolumn{4}{|l|}{ Aggregation-collagen } \\
\hline Collagen 2-Amax (\%) & $74.5-87.3$ & 57.9 & 79.9 \\
\hline Collagen 5-Amax (\%) & $74.7-88.9$ & 72 & 75.8 \\
\hline \multicolumn{4}{|l|}{ Aggregation-ADP } \\
\hline ADP 5-Amax (\%) & $57.0-86.2$ & 57.5 & 69.2 \\
\hline ADP 5-disaggregation (\%) & $0-10.0$ & & \\
\hline ADP 10-Amax (\%) & $66.6-90.7$ & 64.1 & 69.1 \\
\hline ADP 10 -disaggregation (\%) & $0-10.0$ & & \\
\hline \multicolumn{4}{|l|}{ Aggregation-ristocetin } \\
\hline Ristocetin-Amax (\%) & $77.8-97.1$ & 60.6 & 84.9 \\
\hline Ristocetin correction-Amax $(\%)$ & N.A. & & 36.4 \\
\hline Low ristocetin-Amax $(\%)$ & $0.0-10.0$ & 1.0 & 2.6 \\
\hline \multicolumn{4}{|l|}{ Aggregation-arachidonic acid } \\
\hline Arachidonic acid-Amax (\%) & $73.2-89.6$ & 4.7 & 75.0 \\
\hline Spontaneous aggregation & less than 5 & N.D. & N.D. \\
\hline
\end{tabular}

The reference limits were determined based on the results of the 2.5-97.5 percentile. Reference aggregation limits were obtained from 50 peripheral blood donors. Not applicable N.A., not done N.D.

\subsection{TEM Confirmed Macrothrombocytopenia}

TEM confirmed the presence of macrothrombocytes (up to $4.0 \mu \mathrm{m}$ in diameter) with an increased number of $\alpha$-granules of different shapes in patient II- 1 compared with the standard number of $\alpha$-granules in the healthy individual III-3 (Figure 4). A histogram showing the number of $\alpha$-granules per platelet was computed from 10 platelets (Figure 5). Mean and coefficient of variation (CV) were $25.2 \pm 0.36$ for patient II- 1 and $20.3 \pm 0.07$ for healthy individual III-3; Mann-Whitney test $(p<0.045)$. Moreover, greater variability in the $\alpha$-granules number was present in the II- 1 platelets.

\subsection{Immunoblot Analysis}

Immunoblot of platelets confirmed decreased expression of GPIb $\alpha$ in patient II- 1 (0.74) compared with the healthy individual III-2 (1.0) (Figure 6). 

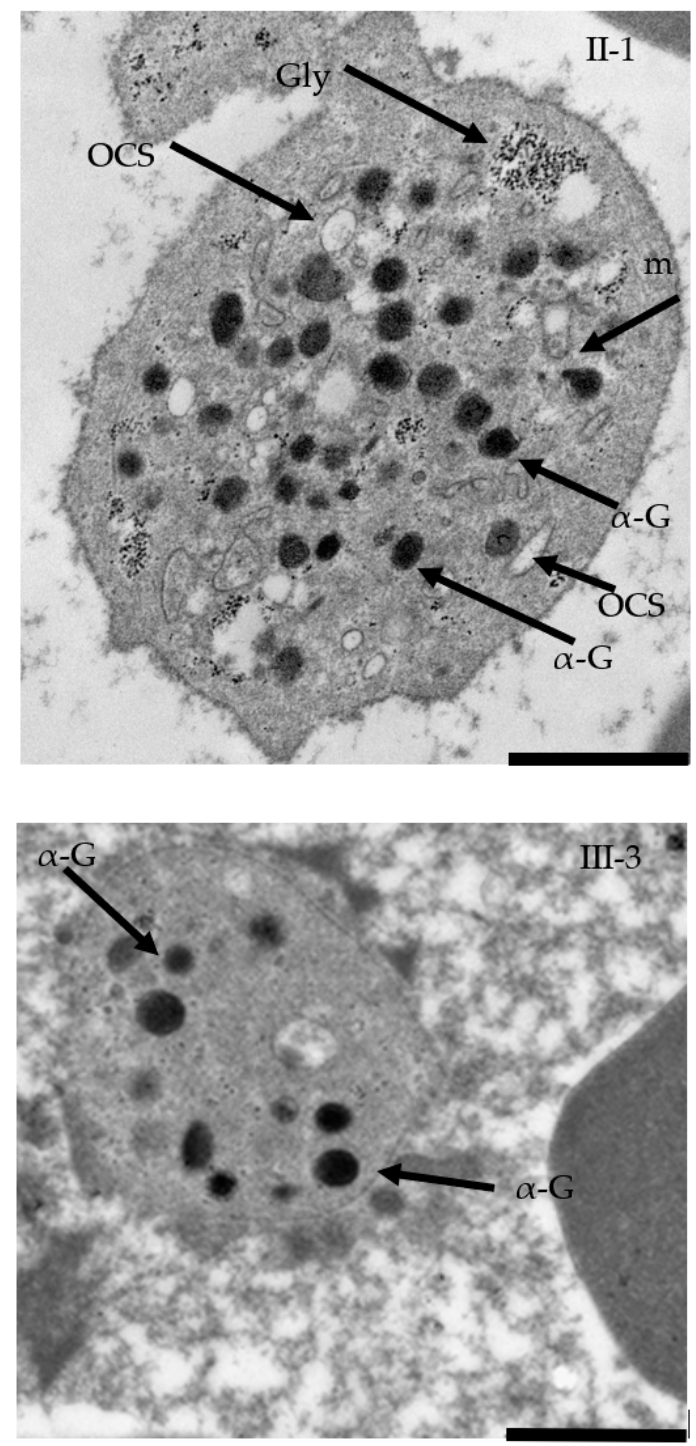

Figure 4. Transmission electron microscopy of a platelet from the affected individual (II-1) and the healthy individual (III-3) platelets. The platelet of the affected individual II-1 is enlarged, containing an increased number of $\alpha$-granules ( $\alpha-\mathrm{G}$ ) of different shapes. The surface-connected open canalicular system (OCS) and an intact mitochondrion ( $\mathrm{m}$ ) and glycogen (Gly) are also seen. Scale bar (a black bar bottom right) represents $1 \mu \mathrm{m}$.
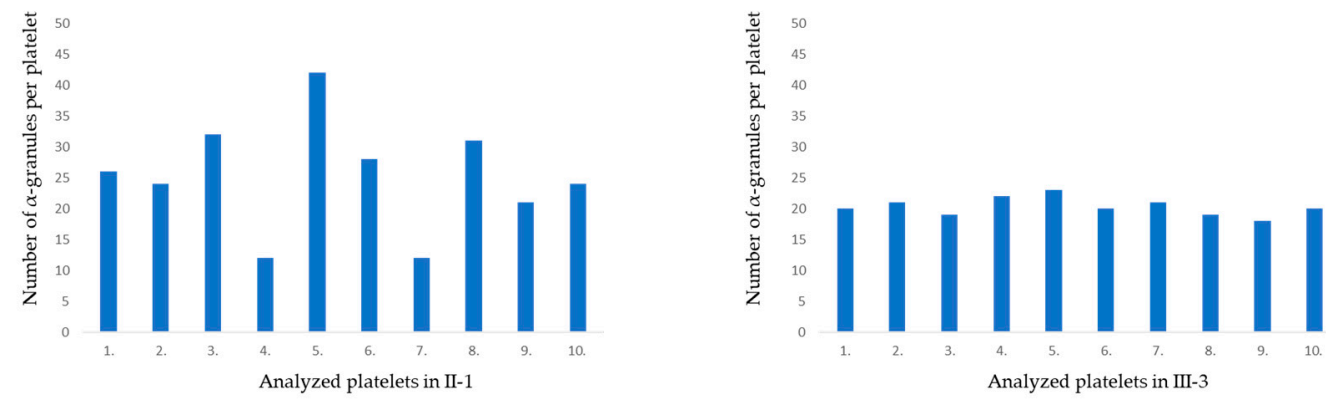

Figure 5. Histograms showing number of $\alpha$-granules per platelet in patient II- 1 and healthy individual III-3. 


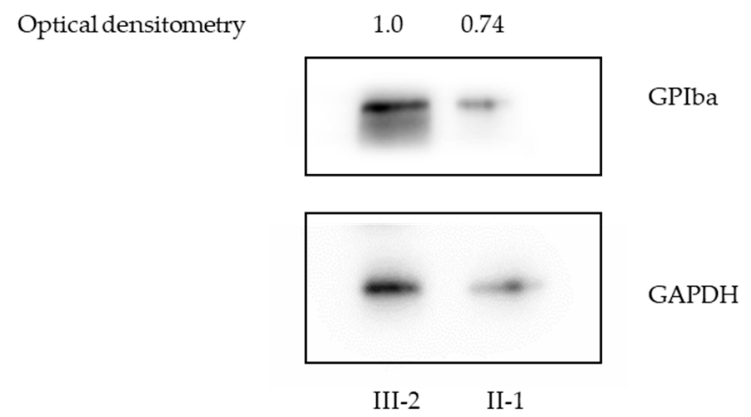

Figure 6. Immunoblot analysis of platelets proteins of patient II-1 and healthy individual III-2 showed decreased expression of GPIb $\alpha 1$ ba in patient II-1 (0.74) compared with healthy individual III-2 (1.0). Antibody GPIba, Rabbit (1:3000), Sigma-Aldrich, antibody GAPDH, Rabbit (1:3000), Cell Signaling.

\section{Discussion}

In this report, we present a family with macrothrombocytopenia and mild bleeding tendency. We report a germline heterozygous variant c.98G > A leading to substitution of p.C33Y in the GP1BA gene, segregating with macrothrombocytopenia in two generations. A causal heterozygous variant c.98G > A of mBSS was described by Leinøe et al. [10] in three patients with moderate thrombocytopenia $\left(81-90 \times 10^{9} / \mathrm{L}\right)$ and undefined macrothrombocytopenia. However, at the same amino acid position was detected the nucleotide variant c.97T > A (p.C33R) in a Chinese family with severe thrombocytopenia $\left(31 \times 10^{9} / \mathrm{L}\right)$, giant platelets, and normal platelet aggregation [11]. Furthermore, we have combined routine laboratory examination of patients with state-of-the-art genomic, flow cytometric, immunoblot, and microscopic approaches to understand the impact of the previously undescribed variant on the patients' phenotype. More than 60 genetic variants in GP1BA have been associated with the classic AR BSS, but to date, only 14 variants have been associated with its AD form. Except for the p.A172V (Bolzano) and p.C20G (Copenhagen) variant $[4,5,10]$, these variants were unique and family specific. Interestingly, all of these variants encoded amino-acid substitution within the LRR region of the GPIb $\alpha$ protein, exerting a dominant-negative effect on the protein structure. Previously described variants affect amino-acid residues deep within the protein structure, disrupting the folding of the LRR region and thus the formation of the GPIb-IX-V complex [1,3-10,13-15,20]. In the case of the here analyzed family, the detected variant substitutes cysteine residue for tyrosine in the conserved N-terminal flanking domain. This results in disruption of the Cys20-Cys33 disulfide bond, which stabilizes terminal LRRs within the arc structure. The importance of Cys33 for LRRs stability supports our hypothesis of the AD mode of inheritance of the p.C33Y variant. Using various in silico online prediction tools, we suggested the likely pathogenic effect of the discovered variant.

The decreased CD9 platelet expression results in the BSS patients are in concordance with the results from the Brazilian BSS patient' cohort published by Beltrame et al. [21], where decreased expression of CD9 together with GPIb $\alpha$ and GPIX in BSS was first described. Decreased CD9 platelet expression in patients with BSS has also been described by Qiao et al. [22]. The observation that CD9 and GPIIb-GPIIIa are stored in the same intracellular structures and migrate to the same activation zones after platelet stimulation supports the previous suggestion of a close association between CD9 and GPIIb-GPIIIa in human platelets and of the possible involvement of CD9 in the adhesive functions of platelets [21]. It is hypothesized that $\mathrm{CD} 9$ modulates integrin $\alpha \mathrm{Ilb} \beta 3$, the major platelet integrin involved in thrombus stability. CD9 also regulates platelet activation and aggregation and cell adhesion [23].

First platelets aggregation of the patient II- 2 from 2017 confirmed BSS diagnosis with severe hypoaggregation after ristocetin and only mildly impaired aggregation after other agonists (ADP, collagen, ARA). New platelet aggregation tests of the proband revealed hypoaggregation $(60 \%)$ after ristocetin $(1.5 \mathrm{mg} / \mathrm{mL})$; however, there was also significant hypoaggregation after other platelet agonists: ADP, collagen, and ARA. The results of 
these tests were affected by the ASA therapy in the proband. Another explanation for the observed hypoaggregation after all aggregation agonists in patients II-1 and II-2 could be the setting of reference limits, which were determined from a normal platelet count in PRP (150-350), while the platelet count in patients II-1 and II-2 were 105 and 102 for these tests. Clinical presentation of BSS patients is heterogeneous: some BSS patients have mainly macrothrombocytes with normal platelet function and may or may not have thrombocytopenia [24]. In addition, in patients who have thrombocytopenia, platelet aggregation may be altered [25]. In our case, we confirmed the monoallelic BSS phenotype in the patient (II-1) based on the platelet aggregation test.

We observed morphological differences of platelets between the affected and healthy family members. We confirmed the presence of macrothrombocytes with an increased number of $\alpha$-granules $(\alpha-G)(25.2 \pm 0.36)$ of different shapes in the II- 1 patient (Figure 4$)$ compared to the healthy individual III-3 $(20.3 \pm 0.07)$. Moreover, our data also demonstrated greater variability in $\alpha$-granule numbers between samples II- 1 and III- 3 , which further supports our hypothesis of likely pathogenic variants. $\alpha$-granules are essential to normal platelet activity. These unusual secretory granules derive their cargo from both regulated secretory and endocytic pathways in megakaryocytes. Furthermore, defects in $\alpha$-granule formation have been described in some inherited disorders of $\alpha$-granules formation, but not in the BSS diagnosis. Functional roles of platelet $\alpha$-granules are derived from their contents and are associated with platelet adhesion. Components of the VWF complex, GPIb $\alpha$-IX-V were found in $\alpha$-granules [26]. The increased number of $\alpha$-granules in this presented case might be explained by the supposition that the GPIb $\alpha$ protein is expressed but does not reach the platelet surface. However, the precise mechanism remains unclear.

Immunoblot of platelets revealed decreased expression of the GPIb $\alpha$ protein in proband II-1 compared with the healthy individual (III-2). This abnormality of the expression of glycoprotein $\mathrm{Ib} \alpha$ of the p.C33Y variant confirms the likely pathogenic effect of the discovered variant.

According to Leinøe et al. [10], the GPIb $\alpha-\mathrm{IX}-\mathrm{V}$ receptor membrane complex plays a pivotal role in thrombosis. Thus, a reduction in the expression of GPIb $\alpha-I X-V$ in mBSS patients may protect against arterial thrombosis and thereby promote a survival advantage. Here, we report the mBSS patient (II-1) with two previous ischemic strokes despite heterozygous GP1BA variant and reduction of GPIb $\alpha-\mathrm{IX}-\mathrm{V}$ complex expression. (Mother (I-2) of this patient had five ischemic strokes before she died, but the GP1BA status is due to the lack of DNA sample unknown). In the here presented patient II-1, the reduced expression of GPIb $\alpha-\mathrm{IX}-\mathrm{V}$ seems not to be protective enough against arterial thrombosis, but other cases are needed for a general conclusion.

In conclusion, the described GP1BA gene variant causes changes at the protein level, which may affect its protein conformation, expression, and thereby disturb the binding to its interacting partners and cause macrothrombocytopenia with decreased expression of GPIb $\alpha$, GPIIa, GPIIIb, CD9 in this case of monoallelic BSS (AD macrothrombocytopenia). Our report also points to the complexity and difficulty of functional analyses and their interpretation in similar cases.

\section{Materials and Methods}

\subsection{Study Approval}

Patients were recruited from University Hospital Brno after written informed consent was obtained, in accordance with the Declaration of Helsinki and protocols approved by the institutional ethics committees.

\subsection{Mutational Screening}

We performed whole-exome sequencing of four family members (II-1, II-2, III-2, III3 ), as shown in Figure 1. Samples of peripheral blood were collected and processed for genomic DNA isolation using the MagCore ${ }^{\circledR}$ Genomic DNA Whole Blood Kit (RBC 
Bioscience, UK). Whole-exome libraries were processed using the KAPA Hyper Prep Kit, SeqCap EZ MedExome Enrichment Kit, and HyperCap Bead Kit (Roche, Pleasanton, CA, USA) according to the SeqCap EZ HyperCap Workflow v2.1 following the recommended protocols. Paired-end 2x75 bp sequencing was performed on the Illumina NextSeq 500 Sequencer (Illumina Inc., San Diego, CA, USA). The sequencing data met the set QC standards for our department: $90 \%$ of reads were mapped to the regions of interest, which had a coverage $\geq 30 \times$. The FastQC tool was applied for quality checks of the sequenced samples. The raw sequencing reads were aligned to the GRCh38 reference genome using BWA-mem, version 0.7.15, including polymerase chain reaction duplicate marks. Germline single nucleotide variants (SNV) and indels were detected via local re-assembly of haplotypes in the GATK HaplotypeCaller, version 3.7. Annotation of the obtained variants/indels was performed with Annovar. The processed SNV/indels were compared first in a panel of 363 platelet-associated genes (Table S4) and then matched to the variants within a whole exome [27]. To identify clinically relevant SNV/indels, only variants with total coverage of at least $15 \times$, minor allele frequency $(\mathrm{MAF})$ values $\leq 0.01$, and predicted possible-probable deleteriousness were included.

Finally, the classification of the characterized variant p.C33Y in the GP1BA gene was determined and called using the consensus guidelines as set out by the American College of Medical Genetics and Genomics and the Association for Molecular Pathology (ACMG/AMP guidelines). The segregation of the p.C33Y variant in four family members was determined by Sanger sequencing using a BigDye Terminator v3.1 Cycle Sequencing Kit according to the manufacturer's protocol. The primers were designed for the detection of exon 2 in the GP1BA gene (F-primer: AGGGGGATCCACTCAAGGC, R-primer: GTCCCATCGACCTGGAGC). Capillary sequencing was performed using BigDye-terminator chemistry on the 3500 Genetic Analyzer (Applied Biosystems, Warrington, UK) [28-33].

\subsection{In Silico Analysis}

The deleterious effect of the p.C33Y was evaluated using in silico online prediction tools according to Table 2. In addition, the crystal structure [20] of the N-terminal domain of human platelet receptor glycoprotein $\mathrm{Ib}$-alpha (GP Ib $\alpha$, pdb code $1 \mathrm{p} 9 \mathrm{a})$ was analyzed using the VMD program [34] to better understand the mutation's effect at the protein structure level.

Table 2. In silico online prediction tools.

\begin{tabular}{cccc}
\hline Tool & Link & Score and Limits & Interpretation \\
\hline Align GVGD & http://agvgd.hci.utah.edu/agvgd_input.php & C65 & "most likely pathogenic" \\
\hline MetalR & google.com/site/jpopgen/dbNSFP & $0.9997(>1.0)$ & "to be deleterious" \\
\hline MutationTaster & http://www.mutationtaster.org & $1.00(\geq 0.46)$ & “disease-causing" \\
\hline PROVEAN & http://provean.jcvi.org/index.php & $-10.07(\leq-2.5)$ & "deleterious effect" \\
\hline SIFT & http://provean.jcvi.org/index.php & $0.001(\leq 0.78)$ & "to be damaging" \\
\hline
\end{tabular}

\subsection{Platelet Immunophenotype}

Peripheral blood samples of the affected patients (II-1, II-2) and a healthy individual (III-3) were collected into EDTA-coated tubes, and PRP was prepared by centrifugation. PRP samples were stained with a single or tricolor combination of fluorochromes. Monoclonal antibodies included CD9: a member of the tetraspanin superfamily (M-L13, Becton, Dickinson and Company, Franklin Lakes, NJ, USA), CD41:GP IIb (VIPL3, Invitrogen), CD42a:GP IX (SZ1, Beckman Coulter), CD42b:GP Ib $\alpha$ (MB45, Invitrogen, ThermoFisher Scientific, Waltham, MA, USA), and CD61:GP IIIa (PM6/13, Fitzgerald Industries International, Acton, MA, USA). An unlabeled negative control sample (native PRP sample) was also prepared. After incubation with antibodies, the samples were analyzed on the flow cytometer BD FACS CANTO II (Becton Dickinson, Franklin Lakes, NJ, USA). 


\subsection{Platelet Aggregation Tests}

In vitro platelet aggregation was studied in citrated platelet-rich plasma. The plateletrich plasma was obtained by centrifugation of blood using the standard technique (at $200 \mathrm{~g}$ for $10 \mathrm{~min}$ ). The platelet agonists were: collagen $2 \mu \mathrm{g} / \mathrm{mL}$ and $5 \mu \mathrm{g} / \mathrm{mL}$ (Hyphen BioMed, Neuville-sur-Oise, France), adenosine diphosphate (ADP, Hyphen BioMed, Neuville-sur-Oise, France) $5 \mu \mathrm{mol} / \mathrm{L}$ and $10 \mu \mathrm{mol} / \mathrm{L}$, ristocetin (Hyphen BioMed, Neuvillesur-Oise, France) $1.5 \mathrm{mg} / \mathrm{mL}$ and low $0.75 \mathrm{mg} / \mathrm{mL}$, arachidonic acid (ARA, Hyphen BioMed, Neuville-sur-Oise, France) $1.5 \mathrm{mmol} / \mathrm{L}$ at a final concentration. We performed aggregation tests on two family members (II-1 and III-2). The reference limits and maximum aggregation results in patients II- 1 and III-2 were obtained (Table 1, Table S5). Reference aggregation limits were obtained from 50 blood donors. The reference limits were determined based on the results of the 2.5-97.5 percentile.

\subsection{Electron Microscopy}

TEM was used to analyze ultrastructural changes in platelets in our family: Venous blood drawn from the patients was mixed with acid citrate dextrose anticoagulant in a 6 to 1 ratio and PRP was prepared by centrifugation at $150 \mathrm{~g}$ for $20 \mathrm{~min}$ at room temperature. After fixation in 3\% glutaraldehyde in phosphate buffer, the platelets were washed with phosphate buffer. Post fixation with 1\% osmium tetroxide was performed and the specimens were dehydrated in a graded series of alcohol and embedded in LR white acrylic resins. Ultrathin serial sections were cut on a Leica Ultracut $\mathrm{R}$ ultramicrotome (Leica, Wetzlar, Germany), stained with uranyl acetate and lead citrate, and examined by a FEI Tecnai G2 F20 microscope (ThermoFisher Scientific, Waltham, MA, USA).

\subsection{Immunoblotting}

Peripheral blood samples of the affected patient (II-1) and the healthy individual (III-2) were collected into sodium citrate coated tubes, platelet isolation was prepared by the density gradient centrifugation and washing method [35]. The platelets sample of the affected patient (II-1) contained: 2.3\% leukocytes (CD45), 84.0\% thrombocytes (CD41, CD61, CD9), and the healthy individual (III-2) contained: $0.86 \%$ leukocytes and $94.3 \%$ of thrombocytes (CD41, CD61, CD9) according to flow cytometric analysis. Immunoblotting of platelets proteins of patient II- 1 and the healthy individual III-2 were diluted with TrisSDS buffer (5 mM Tris, 1\% SDS, 10\% glycerol) and proceeded the same as follows: $10 \mu \mathrm{g}$ of protein lysates were loaded per well. The samples were resolved on 10\% SDS-PAGE and blotted to the PDVF membrane. The membranes were labeled with anti-GPIba (1:3000), $\mathrm{Rb}$, Sigma, anti-GAPDH (1:3000), Rb, Cell Signaling antibodies combined with the Clarity Western ECL Substrate (Bio-Rad, Hercules, CA, USA). The chemiluminescence image of proteins was acquired on the UVITEC CAMBRIDGE system. Optical densitometry of protein bands was calculated using Fiji (ImageJ v.1.53c, open-source software).

Supplementary Materials: The following are available online at https:/ / www.mdpi.com/article/10 $.3390 /$ ijms23020885/s1.

Author Contributions: Conceptualization, M.S. and K.S.K.; data curation, M.S., Z.V. and O.S.; formal analysis, Z.V., L.R., T.K., B.D. and K.R.; investigation, M.S., K.S.K., Z.V., J.Š., M.P. and L.K.; methodology, M.S., Z.V., R.H., O.S., H.S., L.K., M.P. and I.B., project administration, H.S. and B.D.; resources, M.D. and Š.P.; software, L.R.; supervision, Š.P., P.S. and M.D.; validation, M.S., K.S.K. and L.R.; visualization, J.T. and B.D.; writing—original draft, M.S. and K.S.K.; writing-review and editing, J.T., H.S., M.Š., K.R., P.S. and M.D. All authors have read and agreed to the published version of the manuscript.

Funding: This work was supported by the [Ministry of Health of the Czech Republic], under Grant [NU20-08-00137]; [Masaryk University] under Grants [MUNI/A/1330/2021; MUNI/11/SUP/22/2020]; [European Regional Development Fund-Project “A-C-G-T"] under Grant [CZ.02.1.01/0.0/0.0/16_026/ 0008448]. The R\&D division of Thermo Fisher Scientific Brno is greatly acknowledged for allowing access to the Helios FIB-SEM microscope. We acknowledge the Cryo-electron microscopy and 
tomography core facility of CIISB and CEITEC, the Instruct-CZ Centre, supported by MEYS CR [LM2018127]. We acknowledge the CF Genomics CEITEC MU supported by the NCMG research infrastructure [LM2018132 funded by MEYS CR].

Institutional Review Board Statement: The study was conducted according to the guidelines of the Declaration of Helsinki, and approved by the Ethics Committee of the University Hospital Brno (protocol code 22-120619/EK, date of approval: 12 June 2019).

Informed Consent Statement: Informed consent was obtained from all subjects involved in the study.

Data Availability Statement: All data presented in this study are contained within this article or its supplementary materials. Further details are available upon request from the corresponding author.

Acknowledgments: We acknowledge Tomáš Loja for flow cytometric analysis of thrombocytes and Marek Borský and Jana Kotašková for flow cytometric histogrames.

Conflicts of Interest: The authors declare no conflict of interest.

\section{References}

1. Savoia, A.; Kunishima, S.; De Rocco, D.; Zieger, B.; Rand, M.L.; Pujol-Moix, N.; Caliskan, U.; Tokgoz, H.; Pecci, A.; Noris, P.; et al. Spectrum of the Mutations in Bernard-Soulier Syndrome. Hum. Mutat. 2014, 35, 1033-1045. [CrossRef]

2. Kurokawa, Y.; Ishida, F.; Kamijo, T.; Kunishima, S.; Kenny, D.; Kitano, K.; Koike, K.A. Missense mutation (Tyr88 to Cys) in the platelet membrane glycoprotein $\mathrm{Ib} \beta$ gene affects GPIb/IX complex expression. Thromb. Haemost. 2001, 86, 1249-1256. [CrossRef] [PubMed]

3. Kunishima, S.; Naoe, T.; Kamiya, T.; Saito, H. Novel heterozygous missense mutation in the platelet glycoprotein $\mathrm{Ib} \beta$ gene associated with isolated giant platelet disorder. Am. J. Hematol. 2001, 68, 249-255. [CrossRef]

4. Noris, P.; Perrotta, S.; Bottega, R.; Pecci, A.; Melazzini, F.; Civaschi, E.; Russo, S.; Magrin, S.; Loffredo, G.; Di Salvo, V.; et al. Clinical and laboratory features of 103 patients from 42 Italian families with inherited thrombocytopenia derived from the monoallelic Ala156Val mutation of GPIb (Bolzano mutation). Haematologica 2012, 97, 82-88. [CrossRef] [PubMed]

5. Kunishima, S.; Lopez, J.A.; Kobayashi, S.; Imai, N.; Kamiya, T.; Saito, H.; Naoe, T. Missense mutations of glycoprotein (GP) Ib beta gene impairing the GPIb alpha/beta disulfide linkage in a family with giant platelet disorder. Blood 1997, 89, $2404-2412$. [CrossRef] [PubMed]

6. Sivapalaratnam, S.; Westbury, S.K.; Stephens, J.C.; Greene, D.; Downes, K.; Kelly, A.M.; Lentaigne, C.; Astle, W.J.; Huizinga, E.G.; Nurden, P.; et al. Rare variants in GP1BB are responsible for autosomal dominant macrothrombocytopenia. Blood 2017, 129, 520-524. [CrossRef]

7. Savoia, A.; Balduini, C.L.; Savino, M.; Noris, P.; Del Vecchio, M.; Perrotta, S.; Belletti, S.; Poggi, V.; Iolascon, A. Autosomal dominant macrothrombocytopenia in Italy is most frequently a type of heterozygous Bernard-Soulier syndrome. Blood 2001, 97, 1330-1335. [CrossRef]

8. Ghalloussi, D.; Saut, N.; Bernot, D.; Pillois, X.; Rameau, P.; Sébahoun, G.; Alessi, M.C.; Raslova, H.; Baccini, V. A new heterozygous mutation in GP1BA gene responsible for macrothrombocytopenia. Br. J. Haematol. 2018, 183, 503-506. [CrossRef]

9. Trizuljak, J.; Kozubík Staňo, K.; Radová, L.; Pešová, M.; Pál, K.; Réblová, K.; Stehlíková, O.; Smejkal, P.; Zavřelová, J.; Pacejka, M.; et al. A novel germline mutation in GP1BA gene N-terminal domain in monoallelic Bernard-Soulier syndrome. Platelets 2018, 29, 827-833. [CrossRef]

10. Leinøe, E.; Brøns, N.; Rasmusses, A.Ø.; Gabrielaite, M.; Zaninetti, C.; Palankar, R.; Zetterber, E.; Rosthoj, S.; Rye Ostrowski, S.; Rossing, M. The Copenhagen founder variant GP1BA c.58 T>G is the most frequent cause of inherited thrombocytopenia in Denmark. J. Thromb. Haemost. 2021, 19, 2884-2892. [CrossRef]

11. Ma, J.; Chen, Z.; Li, G.; Gu, H.; Wu, R. A novel mutation in GP1BA gene in a family with autosomal dominant Bernard Soulier syndrome variant: A case report. Exp. Ther. Med. 2021, 21, 360-364. [CrossRef] [PubMed]

12. Minkov, M.; Zeitlhofer, P.; Zoubek, A.; Kager, L.; Panzer, S.; Haas, O.A. Novel Compound Heterozygous Mutation in Two Families with Bernard-Soulier Syndrome. Front. Pediatrics 2021, 8, 1-8. [CrossRef] [PubMed]

13. Vettore, S.; Scandellari, R.; Moro, S.; Lombardi, A.M.; Scapin, M.; Randi, M.L.; Fabris, F. Novel point mutation in a leucine-rich repeat of the GPIb chain of the platelet von Willebrand factor receptor, GPIb/IX/V, resulting in an inherited dominant form of Bernard-Soulier syndrome affecting two unrelated families: The N41H variant. Haematologica 2008, 93, 1743-1747. [CrossRef] [PubMed]

14. Miller, J.L.; Lyle, V.A.; Cunningham, D. Mutation of leucine-57 to phenylalanine in a platelet glycoprotein Ib $\alpha$ leucine tandem repeat occurring in patients with an autosomal dominant variant of Bernard-Soulier disease. Blood 1992, 79, 439-446. [CrossRef]

15. Kunishima, S.; Imai, T.; Hamaguchi, M.; Saito, H. Novel heterozygous missense mutation in the second leucine-rich repeat of GPIbalpha affects GPIb/IX/V expression and results in macrothrombocytopenia in a patient initially misdiagnosed with idiopathic thrombocytopenic purpura. Eur. J. Haematol. 2006, 76, 348-355. [CrossRef] 
16. Rodeghiero, F.; Tosetto, A.; Abshire, T.; Arnold, D.M.; Coller, B.; James, P.; Neunert, C.; Lilicrap, D. ISTH/SSC bleeding assessment tool: A standardized questionnaire and a proposal for a new bleeding score for inherited bleeding disorders. J. Thromb. Haemost. 2010, 8, 2063-2065. [CrossRef]

17. Bowman, M.; Mundell, G.; Grabell, J.; Hopman, W.M.; Rapson, D.; Lillicrap, D.; James, P. Generation and validation of the Condensed MCMDM-1VWD Bleeding Questionnaire for von Willebrand disease. J. Thromb. Haemost. 2008, 6, $2062-2066$. [CrossRef]

18. Gresele, P. Diagnosis of inherited platelet function disorders: Guidance from the SSC of the ISTH. J. Thromb. Haemost. 2015, 13, 314-322. [CrossRef]

19. Blenner, M.A.; Dong, X.; Springer, T.A. Structural basis of regulation of von Willebrand factor binding to glycoprotein Ib. J. Biol Chem. 2014, 289, 5565-5579. [CrossRef]

20. Uff, S.; Clemetson, J.M.; Harrison, T.; Clemetson, K.J.; Emsley, J. Crystal structure of the platelet glycoprotein Ib $\alpha$ N-terminal domains reveals an unmasking mechanism for receptor activation. J. Biol. Chem. 2002, 277, 35657-35663. [CrossRef]

21. Beltrame, M.P.; Malvezzi, M.; Zanis, J.; Pasquini, R. Flow cytometry as a tool in the diagnosis of Bernard-Soulier syndrome in Brazilian patients. Platelets 2009, 20, 229-234. [CrossRef] [PubMed]

22. Qiao, J.; Davis, A.K.; Morel-Kopp, M.C.; Ward, C.M.; Gardiner, E.E.; Andrews, R.K. Low levels of CD9 coincidental with a novel nonsense mutation in glycoprotein $\mathrm{Ib} \beta$ in a patient with Bernard-Soulier syndrome. Ann. Hematol. 2015, 94, 2069-2071. [CrossRef]

23. Hill, S.K. The Tetraspanin CD9 Localizes to Platelet-Platelet Contacts and Regulates Thrombus Stability. Ph.D. Thesis, University of Tennessee Health Science Center, Memphis, TN, USA, 2008. [CrossRef]

24. Kunishima, S.; Saito, H. Congenital macrothrombocytopenias. Blood Rev. 2006, 20, 111-121. [CrossRef]

25. Kadlečíková, K. Effect of Quantity of Thrombocytes on Examination of Induced Aggregation of Thrombocytes. Bachelor's Thesis, Masaryk University, Brno, Czech Republic, 2017.

26. Blair, P.; Flaumenhaft, R. Basic biology and clinical correlates. Blood Rev. 2009, 23, 177-189. [CrossRef] [PubMed]

27. Johnson, B.; Lowe, G.C.; Futterer, J.; Lordkipanidze, M.; MacDonald, D.; Simpson, M.A.; Sanchez-Guiu, I.; Drake, S.; Bem, D.; Leo, V.; et al. Whole-exome sequencing identifies genetic variants in inherited thrombocytopenia with secondary qualitative function defects. Haematologica 2016, 101, 1170-1179. [CrossRef] [PubMed]

28. Andrews, S. FastQC: A Quality Control Tool for High Throughput Sequence Data. Available online: https://www.bioinformatics. babraham.ac.uk/projects/fastqc/ (accessed on 9 December 2020).

29. Bolger, A.M.; Lohse, M.; Usadel, B. Trimmomatic: A flexible trimmer for Illumina sequence data. Bioinformatics 2014, 30, 2114-2120. [CrossRef]

30. Okonechnikov, K.; Conesa, A.; García-Alcalde, F. Qualimap 2: Advanced multi-sample quality control for high-throughput sequencing data. Bioinformatics 2015, 32, 292-294. [CrossRef]

31. Smith, T.; Heger, A.; Sudbery, I. UMI-tools: Modeling sequencing errors in Unique Molecular Identifiers to improve quantification accuracy. Genome Res. 2017, 27, 491-499. [CrossRef]

32. Liao, Y.; Smyth, G.K.; Shi, W. FeatureCounts: An efficient general-purpose program for assigning sequence reads to genomic features. Bioinformatics 2014, 30, 923-930. [CrossRef]

33. R Core Team. R: A Language and Environment for Statistical Computing; R Foundation for Statistical Computing: Vienna, Austria. Available online: https:/ / www.r-project.org/ (accessed on 9 December 2020).

34. Humphrey, W.; Dalke, A.; Schulten, K. VMD: Visual Molecular Dynamics. J. Molec. Graph. 1996, 14, 33-38. [CrossRef]

35. Wrzyszcz, A.; Urbaniak, J.; Sapa, A.; Wozniak, M. An efficient method for isolation of representative and contamination-free population of blood platelets for proteomic studies. Platelets 2017, 28, 43-53. [CrossRef] [PubMed] 